\title{
O IMPACTO DA PARTICIPAÇÃO E DA MOTIVAÇÃO NA SATISFAÇÃO COM O TRABALHO: EVIDÊNCIAS DO SETOR PÚBLICO
}

\section{THE IMPACT OF PARTICIPATION AND MOTIVATION ON JOB SATISFACTION: EVIDENCE IN THE PUBLIC SECTOR}

Carina Charpinel Ventorini

Universidade Federal do Espírito Santo

Vitória, ES, Brasil

Email: carinacharpinel@gmail.com

Caroline Ornelas Paes

Universidade Federal do Espírito Santo

Vitória, ES, Brasil

Email: linepaes@gmail.com

\section{Danilo Magno Marchiori}

Universidade Federal do Espírito Santo e Universidade da Beira Interior - NECE (Portugal)

Vitória, ES, Brasil

Email: danilo.marchiori@gmail.com

\section{RESUMO}

Os estudos sobre satisfação no trabalho no setor público, ao identificarem os fatores que nela influenciam, fornecem aos gestores dados para a implantação de melhorias nessa área. Este artigo teve como objetivo investigar o impacto da participação informal e da motivação intrínseca sobre a satisfação no trabalho entre servidores da administração pública federal brasileira, adotando como referências as pesquisas conduzidas por Wang e Yang (2015) e por Cho e Perry (2012). Adicionalmente, verificou, de forma exploratória, se havia diferenças das médias relativas a essas variáveis entre trabalhadores lotados nas unidades descentralizadas e na unidade central. Trata-se de estudo quantitativo e explicativo de corte transversal que utilizou dados secundários da Pesquisa de Clima Organizacional aplicada em um órgão público federal em 2017. Responderam à pesquisa 147 servidores, o que representou uma taxa de resposta de $35 \%$ de uma amostragem não probabilística. Os resultados confirmaram que a participação informal e a motivação intrínseca apresentam influência positiva e estatisticamente relevante sobre a satisfação no trabalho. As análises das médias indicaram que os trabalhadores lotados em unidades descentralizadas possuíam uma percepção de participação informal maior do que os lotados na unidade centralizada.

Palavras-chave: Satisfação no trabalho. Participação informal. Motivação intrínseca. Administração pública. Pesquisa de clima organizacional.

\begin{abstract}
The studies related to job satisfaction in the public sector, as they identify the factors that influence it, provide to managers data for improvements implementation in this area. This article seeks to investigate the impact of informal participation and intrinsic motivation on job satisfaction among Brazilian federal public servants, using as references the researches conducted by Wang and Yang (2015) and by Cho and Perry (2012). In addition, it verified, exploratorily, if there were differences in the averages related to these variables between employees in the decentralized units and in the central unit. This is a cross-sectional quantitative and explanatory study that used secondary data from the Organizational Climate Survey applied at a federal public agency in 2017. The survey was answered by 147 servers, which represented a response rate of $35 \%$ of a nonprobabilistic sample. The results confirmed that informal participation and intrinsic motivation have a positive and statistically relevant influence on job satisfaction. The analysis of the averages indicated that the informal participation perception is greater among the workers located in decentralized units than among the ones located in the centralized unit.
\end{abstract}

Keywords: Job satisfaction. Informal participation. Intrinsic motivation. Public administration.

Organizational climate survey. 


\section{INTRODUÇÃO}

A satisfação no trabalho tem sido extensamente estudada (SPECTOR, 1997), desde o advento da Escola das Relações Humanas, que inaugurou a visão de que uma organização, para ser bem-sucedida, precisa olhar para a satisfação das necessidades dos seus colaboradores (MOTTA; VASCONCELOS, 2004). Em contraste com a expressão 'recursos humanos', anteriormente mais utilizada, as próprias terminologias 'colaborador' e 'gestão de pessoas' retratam esse novo olhar sobre a forma de administrar as organizações e as pessoas que as constituem, o qual surgiu no contexto histórico da referida escola.

Pesquisar a satisfação no trabalho envolve identificar quais variáveis interferem na maneira como as pessoas se sentem em relação ao seu trabalho. A participação informal e a motivação intrínseca são dois fatores de grande relevância que têm sido investigados no contexto do setor público (KIM, 2002; WRIGHT; KIM, 2004; TAYLOR, 2007; TAYLOR, 2008; CHO; PERRY, 2012; TAYLOR, 2014; WANG; YANG, 2015). O estudo realizado por Wang e Yang (2015) com servidores públicos em Beijing, na China, encontrou uma influência positiva e estatisticamente relevante da participação informal em relação à satisfação no trabalho. Já a motivação intrínseca foi pesquisada por Cho e Perry (2012), que constataram a associação desta com a satisfação do empregado no serviço público norte-americano. Ou seja, o tema tem sido estudado no âmbito internacional.

Tais estudos (CHO; PERRY, 2012; WANG; YANG, 2015) foram ponto de partida para a presente pesquisa, que teve como objetivo mensurar o impacto da participação informal e da motivação intrínseca sobre a satisfação no trabalho entre servidores da administração pública federal brasileira. De forma complementar e em caráter exploratório, o trabalho verificou se havia diferenças das médias relativas a essas variáveis entre trabalhadores lotados nas unidades descentralizadas e na unidade central do órgão pesquisado.

Por meio do alcance desse objetivo, podem-se mencionar diversas contribuições que este artigo pretendeu trazer. Em primeiro lugar, buscou-se propiciar avanços na investigação acerca dos fatores em questão que impactam na satisfação no trabalho, avaliando se os dados encontrados por Wang e Yang (2015) e por Cho e Perry (2012) seriam aplicáveis a outros países, como, no caso, o Brasil. É importante destacar que não foram localizados estudos similares neste país, que investigassem os efeitos da participação informal e da motivação intrínseca sobre a satisfação, o que representa uma lacuna que essa pesquisa buscou preencher, trazendo contribuições para a teoria existente, que será melhor abordada no próximo tópico.

Nesse sentido, ressalta-se a importância, para o progresso da ciência, da replicação de pesquisas em contextos diferentes, pois, dessa forma, é possível verificar teorias e buscar elementos que permitam a generalização de resultados (EASLEY; MADDEN; DUNN, 2000; MORRISON; MATUSZEK; SELF, 2010). Vale acrescentar que, no que tange à participação informal, Wang e Yang (2015) indicaram a necessidade de mais pesquisas em outros contextos institucionais e culturais. Por sua vez, Perry, Hondeghem e Wise (2010), ao realizarem revisão da literatura sobre motivação no serviço público no decorrer de 20 anos, concluíram que, em razão de haver ampla difusão de pesquisas nessa área em diversos continentes, há que se considerar que os significados da motivação no serviço público variem de um país para o outro. 
Por fim, a presente pesquisa visou também, ao proporcionar mais conhecimento sobre a área no contexto do serviço público brasileiro, incentivar os gestores públicos a adotarem práticas que possam gerar mais satisfação em suas equipes de trabalho.

\section{REFERENCIAL TEÓRICO}

Serão apresentados, a seguir, fundamentos teóricos e revisão de literatura acerca dos três constructos que foram pesquisados: satisfação no trabalho, participação informal e motivação intrínseca.

\section{Satisfação no trabalho}

A preocupação com a satisfação no trabalho tem sido bastante comum na área de gestão de pessoas, sendo importante uma conceituação sobre o assunto. A definição aqui utilizada diz respeito a quanto as pessoas gostam ou não do seu trabalho (SPECTOR, 1997).

As pesquisas têm analisado a relação de diversas variáveis com a satisfação, o que possibilita às organizações identificar áreas onde há insatisfação e implementar melhorias (SPECTOR, 1997). Ybema, Smulders e Bongers (2010), por exemplo, verificaram a relação entre satisfação e absenteísmo em empresas na Holanda. Traldi e Demo (2012) mostraram a influência do comprometimento organizacional afetivo na satisfação no trabalho e no bem-estar, em pesquisa brasileira com professores de uma universidade federal.

Outra pesquisa abordou a relação entre satisfação no trabalho e saúde física e mental. Por meio de revisão sistemática de literatura e meta análise de 485 estudos, Faragher, Cass e Cooper (2005) encontraram uma indicação de relação altamente significativa entre as referidas variáveis, sendo ainda mais forte no caso da saúde mental (bournout, baixa autoestima, ansiedade e depressão). Diante desses resultados, os pesquisadores recomendaram que as organizações identifiquem os aspectos do trabalho que estejam causando insatisfação.

Dessa forma, promover a satisfação no trabalho trata-se de uma responsabilidade do líder, visando trazer benefícios para a organização e para a sociedade, ao promover a segurança e a saúde ocupacional. (HANTULA, 2015).

Tais considerações apontam para a relevância de se investigar os fatores que afetam a satisfação no trabalho, podendo-se citar, dentre eles, a participação informal e a motivação intrínseca, objeto deste estudo.

\section{Participação informal}

A abordagem teórica adotada, neste artigo, sobre gestão participativa é a formulada por Sashkin (1984), que a define a partir de quatro áreas: estabelecimento de objetivos, tomada de decisões, solução de problemas e realização de mudanças. Segundo o autor, gerir as pessoas de forma participativa traz efeitos positivos para a produtividade, o desempenho e a satisfação no trabalho, pois preenchem as necessidades humanas, quais sejam: autonomia, trabalho significativo e contato interpessoal no ambiente de trabalho.

Sashkin (1984) explica que a participação por meio do estabelecimento de objetivos eleva o desempenho pelos seguintes motivos: o funcionário escolhe objetivos maiores do que o gestor escolheria, o 
que leva a um desempenho maior; a aceitação destes ocorre mais prontamente; constrói-se um clima de confiança e segurança. Como os funcionários sabem mais claramente o que fazer para se chegar aos objetivos, suprem-se as necessidades de autonomia e controle. Por sua vez, a resolução de problemas e a realização de mudanças pelos funcionários aumentam a autonomia e a satisfação em relação a quão significativo é o trabalho. Por fim, a necessidade de contato interpessoal no trabalho é satisfeita pelos métodos participativos em grupos ou equipes. $O$ autor propõe, pois, a gestão participativa como um imperativo ético.

$\mathrm{Na}$ literatura internacional, tem-se encontrado uma relação positiva entre satisfação no trabalho e participação informal. A pesquisa conduzida por Wang e Yang (2015) com servidores civis de Beijing, na China, a qual foi utilizada como referência para a presente pesquisa, indicou que a participação informal afeta positivamente a satisfação no trabalho e que os relacionamentos interpessoais moderam essa relação. A mensuração da participação informal ocorreu a partir do compartilhamento de informações, do envolvimento informal na tomada de decisões e de programas de sugestões informais.

Estudos anteriores já apontavam para essa mesma direção. Kim (2002), em pesquisa realizada em agências governamentais norte-americanas, demonstrou que a gestão participativa e a comunicação efetiva por parte do gestor afetam positivamente a satisfação no trabalho. Posteriormente, em um estudo desenvolvido por Wright e Kim (2004) com funcionários públicos de Nova lorque, os resultados encontrados sugeriram que a tomada de decisão participativa pode contribuir para a elevação da satisfação no trabalho ao influenciar nas características do trabalho, no feedback e no suporte para o desenvolvimento na carreira. Neste caso, vê-se que os autores consideraram como subjacente ao conceito de satisfação no trabalho as características deste, o que remete ao conceito de motivação intrínseca, que será abordado no próximo tópico.

Embora não tenham investigado a satisfação no trabalho, Fernandez e Pitts (2011), ao pesquisarem os fatores que contribuem para comportamentos de inovação em funcionários de agências federais norteamericanas, constataram que o envolvimento dos trabalhadores na tomada de decisões que afetam seu trabalho promoveu a alavancagem da inovação.

$\mathrm{Na}$ literatura brasileira, não foram encontradas pesquisas similares que analisam o impacto da participação sobre a satisfação no trabalho.

\section{Motivação intrínseca}

O conceito de motivação empregado neste artigo diz respeito ao esforço empreendido pelo indivíduo em determinada direção, e envolve tanto a qualidade desse esforço quanto a direção do comportamento (PERRY; PORTER, 1982).

Para compreender o que é motivação intrínseca, faz-se necessária uma explicação conjunta do que é a motivação extrínseca. Para Amabile (1993), a primeira está relacionada à busca, no trabalho, por prazer, interesse, curiosidade, autoexpressão e desafio pessoal, enquanto a segunda refere-se ao engajamento no trabalho para alcançar algum objetivo que não esteja diretamente relacionado ao trabalho, como recompensas, feedback e prazos. Sendo assim, para se fazer essa distinção, analisa-se a forma como a pessoa percebe as tarefas que executa. Segundo a autora, a satisfação no trabalho depende da 
disponibilidade de motivadores intrínsecos e extrínsecos presentes no ambiente de trabalho e de quanto eles combinam com a orientação motivacional de cada indivíduo.

No que diz respeito à área pública, existe o conceito de motivação no serviço público (PSM -public service motivation), que se originou da percepção de que o que motiva os servidores públicos é diferente do que o que motiva os funcionários do setor privado, conforme Perry, Hondeghem e Wise (2010). Tais autores, ao realizarem uma revisão de pesquisas empíricas sobre PSM em um período de 20 anos, observaram que está presente, no cerne do constructo, a ideia de que o comportamento dos servidores públicos orienta-se principalmente para o propósito de fazer o bem aos outros e à sociedade. Dessa forma, a pesquisa constatou que o que há de comum nas várias disciplinas que tratam do tema é que o comportamento humano não é dirigido somente para suas próprias preocupações ou interesses.

A literatura internacional apresenta vários estudos que demonstraram uma associação positiva e significativa entre PSM e a satisfação no trabalho (TAYLOR, 2007; TAYLOR, 2008; CHO; PERRY, 2012; TAYLOR, 2014). Além da satisfação, PSM pode trazer impacto no comprometimento com a organização e na motivação para desempenhar-se bem (TAYLOR, 2007; TAYLOR, 2008). Estudos apontaram também que a burocracia parece reduzir PSM e um ambiente de apoio parece elevá-la (MOYNIHAN; PANDEY, 2007; TAYLOR, 2007).

Ao investigar os efeitos de PSM na satisfação de funcionários federais dos Estados Unidos, Cho e Perry (2012) encontraram uma associação positiva entre essas variáveis e negativa entre PSM e a intenção de deixar o serviço público. Além disso, identificou-se que a confiabilidade do gestor e a diretividade do objetivo são dois fatores que fortalecem essa associação positiva, enquanto a expectativa de recompensas extrínsecas enfraquece essa relação. Anteriormente, Wright (2007) havia também observado resultados semelhantes que apontaram para a atribuição, por funcionários públicos, de uma maior importância em relação aos objetivos da organização e às características da tarefa, gerando um maior impacto na motivação, e de um menor nível de importância às recompensas extrínsecas.

Em pesquisa realizada na China, verificou-se a existência de quatro dimensões de PSM: atração pela elaboração de políticas públicas, compromisso com o interesse público e autossacrifício (LIU; TANG; ZHU, 2008). Estes resultados coadunam-se com a conceituação de PSM citada anteriormente, que se volta para o interesse do servidor em fazer o bem aos outros e à sociedade (PERRY; HONDEGHEM; WISE, 2010). Dessa forma, torna-se importante que os servidores públicos sejam encorajados a perceber que estão contribuindo para o desempenho de um serviço valioso e recebam informações sobre os benefícios proporcionados à sociedade (MOYNIHAN; PANDEY, 2007).

No âmbito do serviço público brasileiro, são raras as pesquisas que tratam de PSM, com exceção da desenvolvida por Buiatti e Shinyashiki (2011), que validaram a escala de PSM com uma amostra no Brasil. Porém, tem-se investigado a motivação e a satisfação entre servidores públicos a partir da teoria dos fatores motivacionais e higiênicos de Herzberg (KLEIN; MASCARENHAS, 2016; BRANDÃO et al, 2014).

Klein e Mascarenhas (2016), por exemplo, pesquisaram como os fatores motivacionais afetam a evasão e a satisfação, na carreira dos gestores governamentais. Os resultados apontaram para a relação da satisfação não somente com fatores extrínsecos, mas principalmente com os intrínsecos, que estão relacionados à natureza do trabalho. Brandão et al (2014) identificaram, na Superintendência Regional do Trabalho e Emprego do Ceará, os fatores preponderantes para satisfação no trabalho e os resultados do 
estudo indicaram como fatores motivacionais a realização profissional, as atividades desempenhadas e a responsabilidade atribuída.

Vale mencionar ainda uma pesquisa brasileira desenvolvida por Rodrigues, Reis Neto e Gonçalves Filho (2014) com 333 funcionários públicos estaduais e municipais de Belo Horizonte, na qual foi observada uma influência direta na motivação no trabalho dos seguintes aspectos: valência da missão institucional, instrumentalidade ligada às recompensas, e autoeficácia no alcance das metas. De forma indireta, influenciaram também na motivação a especificidade e o feedback em relação a metas. Dentre os itens que não exerceram influência, pode se destacar a participação na definição das metas.

\section{Hipóteses e modelo de pesquisa}

Diante do exposto, espera-se que tanto a participação informal quanto a motivação intrínseca (PSM) estejam associadas positivamente à satisfação no trabalho em servidores públicos. Sendo assim, ficam estabelecidas as seguintes hipóteses para a presente pesquisa:

Hipótese 1: A participação informal dos servidores públicos está positivamente relacionada à satisfação no trabalho.

Hipótese 2: A motivação intrínseca dos servidores públicos (PSM) está positivamente relacionada à satisfação no trabalho.

Figura 1: Modelo de pesquisa e hipóteses

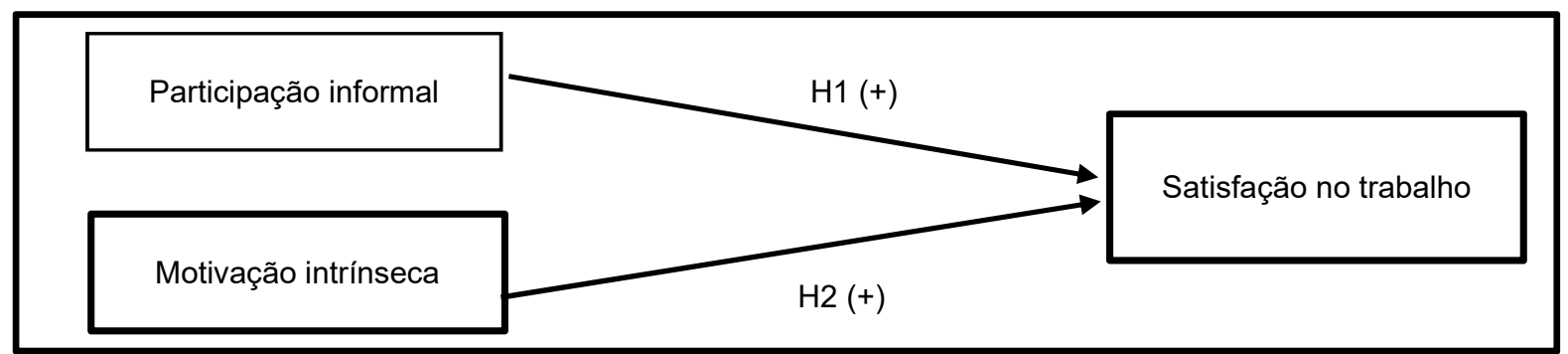

Fonte: elaborada pelos autores

\section{METODOLOGIA}

Esta pesquisa trata-se de um estudo quantitativo e explicativo de corte transversal realizado em um órgão público federal brasileiro. Foram utilizados dados secundários extraídos da Pesquisa de Clima Organizacional aplicada no respectivo órgão em 2017, por meio de formulário eletrônico elaborado no programa Lime Survey e disponibilizado aos servidores pela intranet. Não havia qualquer tipo de identificação dos questionários a fim de preservar o sigilo das informações prestadas. A população da referida instituição era composta por 419 servidores, dos quais 147 responderam à pesquisa, o que representou uma taxa de resposta de $35 \%$, sendo esta uma amostragem não probabilística.

Foram selecionadas da aludida pesquisa as assertivas que correspondiam aos fatores que serão explicados e cujo conteúdo foi analisado como equivalente ao das questões presentes nos estudos adotados como referência (CHO; PERRY, 2012; WANG; YANG, 2015). Os itens utilizados em tais estudos estão 
apresentados no Quadro 1. Gerou-se, assim, um rol de 15 questões, dispostas no Quadro 2, que foram distribuídas da seguinte forma: participação informal (oito itens), motivação intrínseca (quatro itens), e satisfação no trabalho (três itens). Cabe acrescentar que as variáveis independentes deste estudo foram participação informal e motivação intrínseca (PSM) e a variável dependente foi a satisfação no trabalho. No questionário, havia uma escala do tipo Likert com seis opções de respostas relativas ao grau de concordância do servidor com cada assertiva, sendo elas: discordo totalmente, discordo em grande parte, discordo mais do que concordo, concordo mais do que discordo, concordo em grande parte, e concordo totalmente.

\section{Quadro 1 - Itens presentes nos artigos adotados como referência}

\begin{tabular}{|c|l|}
\hline Variáveis & \\
\hline \multirow{2}{*}{$\begin{array}{c}\text { Participação } \\
\text { informal }\end{array}$} & "I get full information of what is going on in my agency". \\
\cline { 2 - 2 } & "I can participate in the decision-making of issues related to my job". \\
\cline { 2 - 2 } Motivação intrínseca & "My supervisors pay attention to my suggestions". \\
\cline { 2 - 2 } & "My work gives me a feeling of personal accomplishment". \\
\cline { 2 - 2 } & "The work I do is important". \\
\hline \multirow{2}{*}{$\begin{array}{c}\text { Satisfação no } \\
\text { trabalho }\end{array}$} & "Considering everything, how satisfied are you with your job?" \\
\cline { 2 - 2 } & "Considering everything, how satisfied are you with your organization?" \\
\cline { 2 - 2 } & "I recommend my organization as a good place to work". \\
\hline
\end{tabular}

Fonte: CHO; PERRY, 2012; WANG; YANG, 2015.

\section{Quadro 2 - Itens selecionados do questionário da Pesquisa de Clima Organizacional}

\begin{tabular}{|c|c|c|}
\hline Variáveis & Sigla & Itens do questionário \\
\hline \multirow{8}{*}{$\begin{array}{l}\text { Participação } \\
\text { informal }\end{array}$} & PAR01 & Tenho autonomia para realizar meu trabalho. \\
\hline & PAR 02 & Há transparência nas decisões tomadas pela minha chefia imediata. \\
\hline & PAR03 & $\begin{array}{l}\text { Participo da definição de metas e objetivos relacionados às atividades que executo } \\
\text { no meu trabalho. }\end{array}$ \\
\hline & PAR04 & Minha chefia imediata aceita facilmente as sugestões que dou. \\
\hline & PAR05 & Na minha unidade, as informações fluem livre e adequadamente. \\
\hline & PAR06 & $\begin{array}{l}\text { Neste Tribunal, existem canais efetivos de comunicação que me mantêm informado } \\
\text { sobre o que se passa nesta Justiça Eleitoral. }\end{array}$ \\
\hline & PAR07 & $\begin{array}{l}\text { As mudanças que ocorrem neste Tribunal são comunicadas e esclarecidas aos } \\
\text { servidores. }\end{array}$ \\
\hline & PAR08 & Sou informado (a) sobre as decisões que venham a afetar meu trabalho. \\
\hline \multirow{4}{*}{ Motivação intrínseca } & MOT01 & Gosto do trabalho que realizo. \\
\hline & MOT02 & Vejo sentido nas atividades que faço. \\
\hline & MOT03 & O trabalho que realizo contribui para o alcance dos resultados do Tribunal. \\
\hline & MOT04 & Meu trabalho me dá um sentimento de realização profissional. \\
\hline \multirow{3}{*}{$\begin{array}{l}\text { Satisfação no } \\
\text { trabalho }\end{array}$} & SAT01 & Tenho orgulho de trabalhar neste Tribunal. \\
\hline & SAT02 & Considero este Tribunal um bom lugar para trabalhar. \\
\hline & SAT03 & De forma geral, estou satisfeito (a) em trabalhar neste Tribunal. \\
\hline
\end{tabular}

Fonte: dados da Pesquisa de Clima Organizacional 2017, realizada no órgão estudado. 
Dentre os dados coletados, aqueles relativos a 13 participantes foram eliminados por preenchimento incompleto do questionário, com mais de $5 \%$ de omissões pelo respondente, e três por serem outliers extremos (MARÔCO, 2014), resultando, portanto, em uma amostra de 131 respondentes a ser trabalhada. Deste total, 58 encontravam-se lotados em unidades descentralizadas, fisicamente afastadas da Administração do órgão, e 73 na unidade centralizada. Os demais casos de omissão, que não implicaram na exclusão do respondente, foram substituídos pela média das respostas da referida questão (PASQUALI, 2015).

As análises de dados e testes estatísticos foram realizadas através do programa Statistical Packcage for Social Science 22 (SPSS). Calcularam-se os alfas de Cronbach para cada dimensão estudada, obtendose os valores 0,901 para satisfação no trabalho; 0,820 para motivação intrínseca; e 0,839 para participação informal.

Para a análise dos dados, foram verificados os pressupostos de homocedasticidade e de normalidade. Realizou-se o teste de Levene, que confirmou a homogeneidade da variância, e o teste de Kolmogorov-Smirnov, o qual apresentou desvios no que tange à verificação da normalidade. Ainda que o pressuposto de normalidade tenha sido violado, por se tratar de uma amostra maior que 30 (MARÔCO 2014), os testes paramétricos são robustos a essa violação.

Procedeu-se, portanto, uma regressão linear a fim de verificar e quantificar a interação dos fatores motivação intrínseca e participação informal sobre a satisfação no trabalho. Esta regressão mostrou que a relação entre os fatores estudados é estatisticamente relevante e, através do $R^{2}$ ajustado, observou-se que cerca de $36 \%$ da satisfação no trabalho é explicada por estes fatores. Constatou-se também a não existência de colinearidade entre os fatores, com um VIF de 1,12, sendo menor que 5 (MOLTGOMERY; PECK, 1982). Como condição para a regressão linear, analisaram-se a normalidade e a homocedasticidade dos resíduos através da análise gráfica, que indicou uma distribuição aproximadamente normal e características de homogeneidade da variância.

Adicionalmente à regressão, foi aplicado o Teste-t de amostras independentes para verificar se existia diferença estatística entre as percepções dos servidores lotados na unidade centralizada e nas unidades descentralizadas. Através do teste, constatou-se que apenas no fator participação informal houve diferença relativa à lotação. 


\section{RESULTADOS E DISCUSSÃO}

Nesta seção, serão apresentados os resultados junto à discussão quanto à confirmação ou não das hipóteses.

\section{Estatísticas descritivas}

A Tabela 1 traz as estatísticas descritivas dos fatores analisados.

Tabela 1 - Estatísticas descritivas

\begin{tabular}{lcccc}
\hline & Fator & & Média & Desvio-Padrão \\
\hline \multirow{3}{*}{ Satisfação no trabalho } & Geral & & $\mathbf{5 , 0 8}$ & $\mathbf{0 , 9 2 0}$ \\
& & UD & 5,08 & 0,831 \\
& & UC & 5,07 & 0,991 \\
Motivação intrínseca & Geral & & $\mathbf{4 , 8 6}$ & $\mathbf{0 , 8 6 8}$ \\
& & UD & 4,96 & 0,790 \\
Participação informal & \multirow{3}{*}{ Geral } & UC & 4,78 & 0,923 \\
& & & $\mathbf{4 , 3 9}$ & $\mathbf{0 , 9 1 7}$ \\
& & UD & 4,58 & 0,804 \\
& & UC & 4,24 & 0,976 \\
\hline
\end{tabular}

Nota: a sigla UD foi criada para denominar unidades descentralizadas e UC para unidade centralizada.

Fonte: Elaborada pelos autores (2017).

A partir dos resultados apresentados, destaca-se que os servidores do órgão analisado estão satisfeitos no trabalho que executam, com uma média de 5,08 que representa $81,6 \%$ do valor máximo possível. Semelhante a este fator, conforme se pode observar na Tabela 1, os servidores se sentem motivados, nota média de 4,86 que representa $77,18 \%$ da nota máxima, e têm a percepção de que participam da organização, nota média de 4,39 que representa $67,80 \%$ da nota máxima. 


\section{Regressão múltipla}

Após validada a amostra pelos testes descritos no capítulo 3, realizou-se a regressão linear múltipla entre a variável dependente satisfação no trabalho e as variáveis independentes motivação intrínseca (PSM) e participação informal. $O$ objetivo desta regressão foi verificar e quantificar a relação entre estes construtos e assim confirmar as hipóteses $\mathrm{H}_{1}$ e $\mathrm{H}_{2}$. A Tabela 2 apresenta o resumo dos resultados da estimação do modelo, onde se pode verificar que cerca de $36 \%$ da satisfação no trabalho é explicada pelos preditores apresentados neste estudo.

Tabela 2 - Resumo do modelo

\begin{tabular}{|c|c|c|c|c|c|}
\hline Variável dependente & Preditores & $\mathrm{R}$ & $\begin{array}{c}\mathrm{R} \\
\text { quadrado }\end{array}$ & $\begin{array}{c}\text { R quadrado } \\
\text { ajustado }\end{array}$ & Erro padrão da estimativa \\
\hline Satisfação no trabalho & $\begin{array}{c}\text { Motivação } \\
\text { intrínseca } \\
\text { Participação } \\
\text { informal }\end{array}$ & $0,605^{\mathrm{a}}$ & 0,366 & 0,356 & 0,738 \\
\hline
\end{tabular}

A Tabela 3 traz os coeficientes obtidos por meio da regressão linear múltipla.

Tabela 3 - Coeficientes

\begin{tabular}{cccc}
\hline Modelo & Coeficientes padronizados & Sig & Estatísticas de colinearidade \\
& Beta & & VIF \\
\hline Motivação intrínseca & 0,376 & 0,000 & 1,120 \\
Participação informal & 0,367 & 0,000 & 1,120 \\
\hline
\end{tabular}

Fonte: Elaborada pelos autores (2017).

Assim, os resultados mostraram que as variáveis independentes de fato influenciam direta e positivamente na satisfação no trabalho, sendo que a motivação intrínseca possui um peso ligeiramente maior que a participação informal. 


\section{Comparação de médias}

Com o objetivo de verificar se há diferenças das médias relativas às variáveis estudadas entre trabalhadores lotados nas unidades descentralizadas e na unidade central, realizou-se o Teste-t de amostras independentes, que compara as médias dos grupos - Tabela 4.

\section{Tabela 4 - Resultados Teste-t}

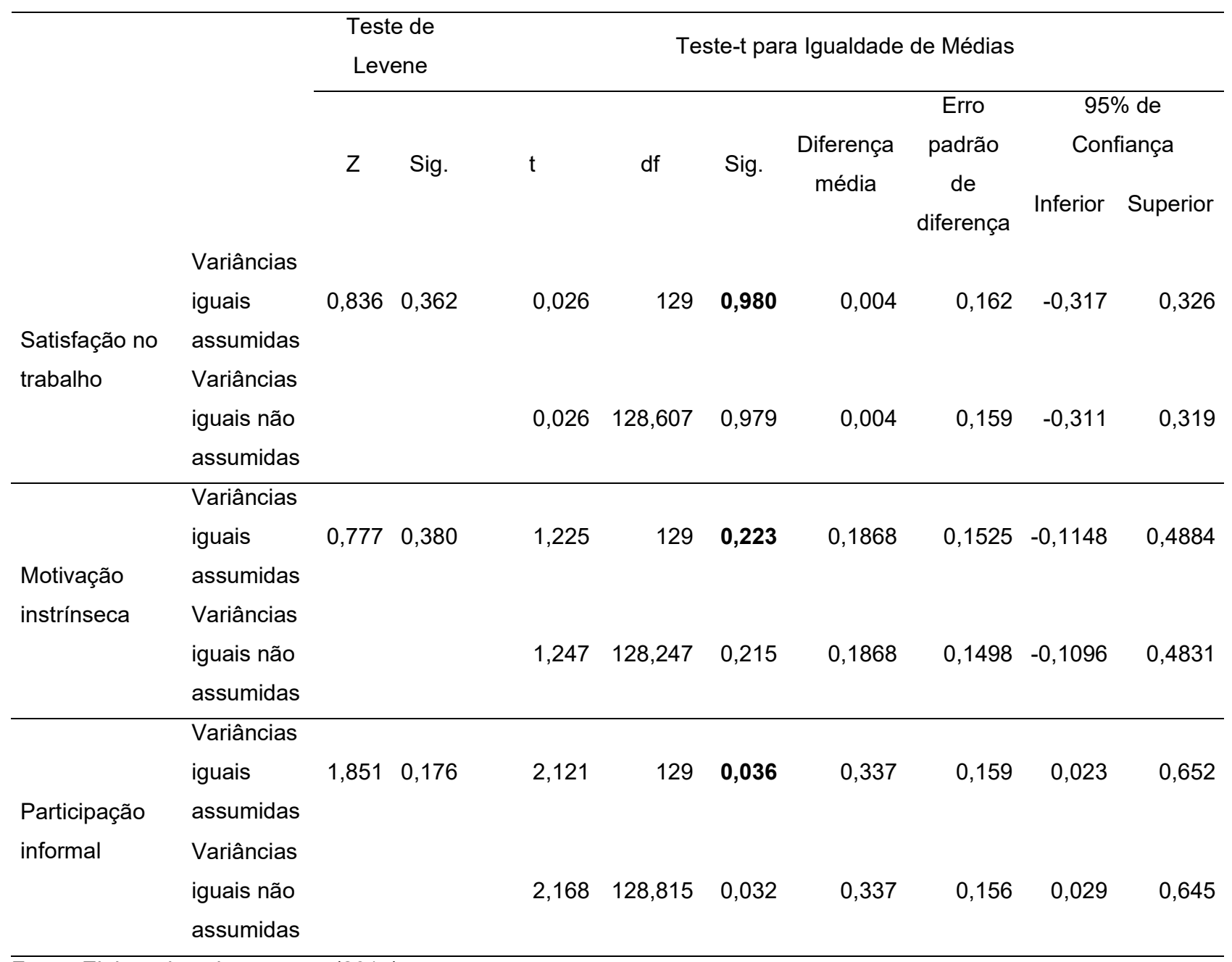

Fonte: Elaborada pelos autores (2017).

Analisando os resultados do Teste-t, observa-se que apenas para a participação informal existe diferença estatisticamente relevante entre as médias. Os servidores que trabalham nas unidades descentralizadas têm a percepção de participação informal maior que os servidores da unidade centralizada. 


\section{Discussão}

Os resultados obtidos na presente pesquisa suportam totalmente as duas proposições apresentadas de que a satisfação no trabalho dos servidores públicos é afetada positivamente pela participação informal e pela motivação intrínseca (PSM).

A primeira hipótese supôs que a participação informal dos servidores públicos está positivamente relacionada à satisfação no trabalho e foi satisfatoriamente confirmada neste trabalho, corroborando com as pesquisas realizadas por Wang e Yang (2015), Kim (2002) e Wright e Kim (2004). Ou seja, promover a participação informal dos servidores públicos em suas organizações parece produzir uma elevação da percepção de satisfação no trabalho, com consequências benéficas para a organização e para o trabalhador, como abordado no capítulo 2 desta pesquisa. Tais resultados podem ser explicados a partir da abordagem teórica de Sashkin (1984), que enunciou que a participação informal, ao satisfazer as necessidades de autonomia, trabalho significativo e contato interpessoal no ambiente de trabalho, promove a produtividade, 0 desempenho e a satisfação no trabalho.

A segunda hipótese indicou que a motivação intrínseca dos servidores públicos (PSM) está positivamente relacionada à satisfação no trabalho, sendo esta proposição também confirmada satisfatoriamente pelas análises estatísticas realizadas, o que vai ao encontro dos estudos conduzidos por Cho e Perry (2012) e Taylor (2007, 2008, 2014). Novamente, vê-se que aumentar a motivação intrínseca percebida pelos servidores públicos impacta positivamente a satisfação no trabalho. Essa motivação, segundo Amabile (1993), ocorre quando o trabalhador se engaja no trabalho, buscando nele prazer, interesse, curiosidade, autoexpressão e desafio pessoal. Assim, é importante que os gestores identifiquem o perfil de competências e de interesses dos servidores e busquem estratégias que promovam motivação intrínseca a fim de melhorar a satisfação, por exemplo delegando atividades que despertem o interesse e a curiosidade do indivíduo.

Vale salientar que, a partir da análise dos resultados obtidos, o fator motivação intrínseca afeta a satisfação no trabalho com intensidade ligeiramente maior que a participação informal. Ou seja, havendo uma situação onde haja necessidade de escolher entre os dois fatores estudados para obter o maior impacto sobre o constructo satisfação no trabalho, a motivação intrínseca seria a mais indicada de acordo com os dados analisados.

Finalmente, os resultados das comparações de médias dos constructos entre as unidades descentralizadas e a unidade centralizada apresentaram diferença com relevância estatística apenas para o constructo participação informal. Os servidores lotados nas unidades descentralizadas demonstraram uma percepção de participação informal maior que os lotados na unidade centralizada. Essa diferença, porém, não foi suficiente para influenciar a variável dependente estudada (satisfação no trabalho), ainda que esta apresente um comportamento compatível com este achado, pois a satisfação nas unidades descentralizadas também é maior que na unidade centralizada. Uma possível explicação é o fato de que, ainda que os constructos estudados tenham relação positiva com a satisfação, estes não são suficientes para determinála completamente.

Tendo em vista o resultado de que a percepção de participação informal é maior nas unidades descentralizadas e que apenas esse constructo apresentou diferença estatisticamente significativa, buscou- 
se uma explicação baseando-se na observação das atividades da organização estudada. Um ponto a ser considerado é que, nas unidades descentralizadas, existe uma menor compartimentalização dos setores, menos níveis hierárquicos e os servidores ficam mais próximos de sua chefia imediata. Assim, acredita-se que essa proximidade entre servidores e chefia bem como a menor compatimentalização e hierarquização contribuem para que as decisões sejam tomadas com maior participação de todos os servidores.

Com caráter exploratório, a fim de confirmar essa suposição, foi realizada uma última análise de comparação de médias. Separaram-se as assertivas que compõem a participação informal em duas partes: (A) assertivas de 01 a 05 que tratam de situações entre o servidor e sua chefia imediata ou o servidor e sua atividade direta; (B) assertivas de 06 a 08 que tratam de situações entre a organização de maneira geral e o servidor. Ao analisar as médias entre unidades centralizada e descentralizadas utilizando esta divisão, verificou-se que, apenas na situação $(A)$, houve diferença significativa entre as unidades descentralizadas e centralizada, o que parece confirmar a suposição inicial. Este resultado, porém, é apenas preliminar e deve ser melhor explorado em pesquisas futuras.

\section{CONCLUSÃO}

Este estudo investigou o impacto da participação informal e da motivação intrínseca sobre a satisfação no trabalho entre servidores da administração pública federal brasileira, adotando como referências as pesquisas conduzidas por Wang e Yang (2015) e por Cho e Perry (2012). Adicionalmente, verificou, de forma exploratória, se havia diferenças das médias relativas a essas variáveis entre trabalhadores lotados nas unidades descentralizadas e na unidade central.

Os resultados encontrados confirmaram as hipóteses formuladas e, portanto, a aplicabilidade dos dados encontrados por Wang e Yang (2015) e por Cho e Perry (2012) ao serviço público brasileiro. As análises das médias indicaram que os trabalhadores lotados em unidades descentralizadas possuíam uma percepção de participação informal maior do que os lotados na unidade centralizada.

Diante disso, uma primeira implicação desta pesquisa para o serviço público refere-se à relevância de práticas de gestão participativa e, principalmente, de promoção da motivação intrínseca a fim de que se eleve a satisfação no trabalho entre servidores públicos. $O$ impacto de tais ações pode se refletir em benefícios para a saúde física e mental da força de trabalho (FARAGHER; CASS; COOPER, 2005), fomentando a segurança e a saúde ocupacional (HANTULA, 2015). Dessa forma, no que concerne à motivação intrínseca, é importante que os gestores públicos viabilizem condições para que os servidores visualizem o impacto positivo dos seus esforços para o bem-estar dos outros (TAYLOR, 2014), expondo não somente o que e como fazer, mas também por que fazer (WRIGHT, 2007). Além disso, mecanismos de participação informal podem ser implementados no cotidiano pelas lideranças do setor público, como tomada conjunta de decisões, compartilhamento de informações e levantamento de sugestões, envolvendo custos mais baixos do que métodos formais de participação (WANG; YANG, 2015).

Outra implicação diz respeito ao planejamento de capacitações direcionadas aos gestores públicos. Ao incluir em seus objetivos o ensino de competências de gestão participativa e de promoção da motivação intrínseca junto à equipe de trabalho, os programas de ensino poderão trazer contribuições significativas para um quadro de servidores mais satisfeitos com o trabalho. 
É importante ressaltar limitações ao presente trabalho. Ressalta-se que o estudo foi desenvolvido a partir de dados referentes a apenas um órgão público federal brasileiro, com uma amostragem não probabilística, o que faz com que os resultados não possam ser generalizados.

Recomenda-se que futuras pesquisas sejam realizadas a fim de explorar as demais variáveis que explicam a satisfação no trabalho e estudar os fatores que moderam a relação entre estas. Além disso, recomendam-se estudos sobre a participação informal em outras organizações com características similares quanto à existência de unidades centralizada e descentralizadas com o intuito de explorar os fatores que influenciam este constructo.

\section{REFERÊNCIAS}

AMABILE, T. M. Motivational synergy: toward new conceptualizations of intrinsic and extrinsic motivation in the workplace. Human Resource Management Review, v. 3, n. 3, p. 185-201, 1993.

BRANDÃO, I. de F. et al. Satisfação no serviço público: um estudo na Superintendência Regional do Trabalho e Emprego no Ceará. Revista Eletrônica de Administração, Porto Alegre, v. 20, n. 1, p. 90-113, 2014. Disponível em: < http://www.scielo.br/pdf/read/v20n1/a04v20n1.pdf>. Acesso em: 28 set. 2017.

BUIATTI, C. L.; SHINYASHIKI, G. T. Gestão de RH no setor público: validação da escala de PSM - public service motivation. Iberoamerican Journal of Industrial Engeneering, Florianópolis, v. 3, n. 1, p.118-139, jul./2011.

$\mathrm{CHO}$, Y. J.; PERRY, J. L. Intrinsic motivation and employee attitudes. Review of Public Personnel Administration, v. 32, n. 4, p. 382-406, 2012.

EASLEY, R. W.; MADDEN, C. S.; DUNN, M. G. Conducting marketing science: the role of replication in the research process. Journal of Business Research, New York, v. 48, n. 1, p. 83-92, 2000.

FARAGHER, E. B.; CASS, M.; COOPER, C. L. The relationship between job satisfaction and health: a meta-analysis. Occupational and Environmental Medicine, v. 62, n. 2, p. 105-112, 2005.

FERNANDEZ, S.; PITTS, D. W. Understanding employee motivation to innovate: evidence from front line employees in united states federal agencies. Australian Journal of Public Administration, v. 70, n. 2, p. 202-222, 2011.

HANTULA, D. A. Job Satisfaction: the management tool and leadership responsibility. Journal of Organizational Behavior Management, v. 35, n. 1-2, p. 81-94, 2015.

KIM, S. Participative management and job satisfaction: lessons for management leadership. Public Administration Review, v. 62, n. 2, p. 231-241, mar./apr. 2002.

KLEIN, F. A.; MASCARENHAS, A. O. Motivação, satisfação profisional e evasão no serviço público: o caso da carreira de especialistas em políticas públicas e gestão governamental. Revista de Administração Pública, Rio de Janeiro, v. $50, n$. 1, p. 17-39, jan./fev. 2016. Disponível em: < http://www.scielo.br/pdf/rap/v50n1/0034-7612-rap-50-01-00017.pdf>. Acesso em: 28 set. 2017.

LIU, B. TANG, N.; ZHU, X. Public service motivation and job satisfaction in China: na investigation generalisability and instrumentality. International Journal of Manpower, v. 29, n. 8, p. 684-699, 2008.

MARÔCO, J. Análise estatística com o SPSS Statistics. 6. ed. Lisboa: Editora Reportnumber, 2014.

MOLTOGOMERY, D. C.; PECK, E. A. Introduction to linear regression analysis. New York: John Wiley \& Sons, 1982.

MORRISON, R.; MATUSZEK, T.; SELF, D. Preparing a replication or update study in the business disciplines. European Journal of Scientific Research, v. 47, n. 2, p. 278-287, 2010.

MOTTA, F. C. P.; VASCONCELOS, I. F. Teoria geral da administração. São Paulo: Thomson Learning, 2004.

MOYNIHAN, D. P.; PANDEY, S. K. The role of organizations in fostering public service motivation. Public Administration Review, v. 67, n. 1, p. 40-53, 2007.

PASQUALI, L. Delineamento de pesquisa em ciência. São Paulo: Editora Vetor, 2015.

PERRY, J. L.; HONDEGHEM, A.; WISE, L. R. Revisiting the motivational bases of public service: twenty years of research and an agenda for the future. Public Administration Review, v. 70, n. 5, p. 681-690, 2010.

PERRY, J. L.; PORTER, L. W. Factors affecting the context for motivation in public organizations. The Academy of Management Review, v. 7, n. 1, p. 89-98, jan. 1982.

RODRIGUES, W. A.; REIS NETO, M. T.; GONÇAVES FILHO, C. As influências na motivação para o trabalho em ambientes com metas e recompensas um estudo no setor público. Revista de Administração Pública, Rio de Janeiro, v. 


\section{O IMPACTO DA PARTICIPAÇÃO E DA MOTIVAÇÃO NA SATISFAÇÃO COM O TRABALHO: EVIDÊNCIAS DO SETOR PÚBLICO}

48, n. 1, p. 253-273, jan. fev. 2014. Disponível em: < http://www.scielo.br/pdf/rap/v48n1/a11v48n1.pdf>. Acesso em: 29 set. 2017.

SASHKIN, M. Participative management is an ethical imperative. Organizational Dynamics, v. 12, p. 5-22, 1984.

SPECTOR, P. E. Job satisfaction: application, assessment, causes, and consequences. Thousand Oaks: Sage, 1997.

TAYLOR, J. The impact of public service motives on work outcomes in Australia: a comparative multi-dimensional analysis. Public Administration, v. 85, n. 4, p. 931-959, 2007.

TAYLOR, J. Organizational influences, public service motivation and work outcomes: an australian study. International Public Management Journal, v. 11, n. 1, p. 67-88, 2008.

TAYLOR, J. Public service motivation, relational job design, and job satisfaction in local government. Public Administration, v. 92, n. 4, p. 902-918, 2014.

TRALDI, M. T. F.; DEMO, G. Comprometimento, bem-estar e satisfação dos professores de administração de uma universidade federal. Revista Eletrônica de Administração, Porto Alegre, v. 72, n. 2, p. 290-316, maio/ago. 2012. Disponível em: < http://seer.ufrgs.br/index.php/read/article/view/38389/24661>. Acesso em: 29 set. 2017.

WANG, W.; YANG, X. Does informal participation increase job satisfaction in public organizations? A study on civil servants in Beijing, China. Public Personnel Management, v. 44, n. 3, p. 356-374, 2015.

WRIGHT, B. E. Public service and motivation: does mission matter? Public Administration Review, v. 67, n. 1, p. 54-64, jan./feb. 2007.

WRIGHT, B. E.; KIM, S. Participation's influence on job satisfaction. Review of Public Personnel Administration, v. 24, n. 1, p. 18-40, 2004.

YBEMA, J. F.; SMULDERS, P. G. W.; BONGERS, P. M. Antecedents and consequences of employee absenteeism: a longitudinal perspective on the role of job satisfaction and burnout. European Journal of Work and Organizational Psychology, v. 19, n. 1, p. 102-124, mar. 2010. 